\title{
Nueva propuesta para la evaluación de la condición física en árbitros de fútbol
}

\author{
New proposal for the physical fitness evaluation in football referees
}

\author{
Carlos Albaladejo Garcia* y Roberto Cejuela Anta \\ Area Educación Física y Deportiva. Departamento Didáctica General y Didácticas Específicas. Universidad de Alicante (España).
}

\begin{abstract}
Resumen: El objetivo de este trabajo era aplicar dos baterías de evaluación de la condición física en árbitros de fútbol no profesionales, en un intento de determinar cuál puede ser más específica para este colectivo. Los árbitros $(n=10)$ fueron evaluados en tres momentos de la temporada (T1, T2, T3), realizando la batería actualmente utilizada por la Federación de Fútbol de la Comunidad Valenciana (FFCV) (RSA: 6x40m/90"; Resistencia: carrera de $2000 \mathrm{~m}$ ) y otra de nueva propuesta (RSA: $7 \times 30 \mathrm{~m} / 25$ ”; Resistencia: Test Interválico para la Valoración de la Resistencia Específica en Fútbol, TIVRE-F). La Velocidad Aeróbica Máxima (VAM) de los árbitros aumentó significativamente en el TIVRE-F entre T1 y T3. El test 7x30/25” registró mayores índices de fatiga que $6 \times 40 / 90$ " en T2 y T3. Con este trabajo se han obtenido nuevos datos sobre las pruebas que la FFCV utiliza en sus árbitros, concluyendo que la propuesta podría ajustarse en mayor medida a las necesidades de evaluación.

Palabras clave: colegiado, test, rendimiento, TIVRE-F, repeated sprint ability, balonpié.
\end{abstract}

Abstract: The aim of this study was the application of two batteries of tests in non-professional soccer referees, trying to determine which of them could be more specific for referees. The participants $(n=10)$ were evaluated in three moments of the season (T1, T2, T3), performing the current battery used by the Football Federation of the Valencian Community (FFCV) (RSA: 6x40m/90"; Endurance: $2000 \mathrm{~m}$ run) and another new proposal battery (RSA: 7x30m/25"; Endurance: Interval Test for Specific Endurance in Football, TIVRE-F). The results showed that the MAV of the referees significantly increased in the TIVRE-F between T1 and T3. The 7x30/25" test reported higher sprint decrement than $6 \times 40 / 90$ " in T2 and T3. From this study we have obtained new data about the current FFCV tests, and we can conclude that the new battery of tests could adjust in a better way to the evaluation necessities.

Keywords: official, test, performance, TIVRE-F, repeated sprint ability, soccer.

\section{Introducción}

A pesar de que la condición física no es el único factor que determina el rendimiento, es un componente muy relevante para el colectivo arbitral (Castillo, Yanci, Casajús, \& Cámara, 2016). Por este motivo, se precisa una acción evaluadora de calidad para comprender el estado de forma de los árbitros a lo largo de la temporada.

Teniendo en cuenta que se han encontrado semejanzas en las demandas físicas y fisiológicas de árbitros y jugadores de fútbol (Castagna, Abt \& D’Ottavio, 2007; Reilly \& Gregson, 2006; Stølen, Chamari, Castagna \& Wisløff, 2005; Weston, Drust \& Gregson, 2011), el entrenamiento y evaluación de la condición física debería seguir las mismas pautas de especificidad.

La Repeated Sprint Ability (RSA) y la cualidad aeróbica son factores influyentes en el rendimiento en fútbol, "tanto para ser capaces de realizar ejercicio intermitente a alta intensidad de forma estable como para optimizar la recuperación minimizando los efectos de la fatiga" (Barbero-Álvarez, 2010). Del mismo modo, también lo son para el rendimiento en árbitros de fútbol (Castagna, Abt, \& D’Ottavio, 2004; Mallo, Navarro, García-Aranda, Gilis \& Helsen, 2007; Krustrup \& Bangsbo, 2001; Krustrup et al., 2009).

Sin embargo, la Fédération Internacionale de Football As-

Dirección para correspondencia [Correspondence address]: Carlos Albaladejo Garcia. E-mail: albala.carlos@gmail.com sociation (FIFA) ha utilizado, durante años, baterías de tests que no se adecúan al perfil de actividad física de los árbitros. En 2005, FIFA introdujo una batería que pretendía evaluar la velocidad media durante esprints repetidos $(6 \times 40 \mathrm{~m}$, con una recuperación de 90 segundos entre repeticiones) y el rendimiento aeróbico en carreras repetidas de alta intensidad (20x150m en 30 segundos, alternados con marcha de $50 \mathrm{~m}$ en 40 segundos).

Mallo, Navarro, García-Aranda y Helsen (2009) encontraron que los resultados obtenidos en estos tests eran pobres predictores del rendimiento dentro del terreno de juego. Una opinión generalizada está encaminada a que estas pruebas no presentan la validez ideal que deberían poseer (Cerqueira, Da Silva, \& Marins, 2011; Schenk, Bizzini, \& Gatterer, 2018; Weston et al., 2012). Estos tests conllevan patrones de carrera que no se ajustan a las actividades a las que los árbitros están sometidos durante los partidos, caracterizadas por su multidireccionalidad. Por tanto, se estaría obviando un punto clave en la evaluación de la aptitud física, que es la especificidad.

Actualmente, el test de $6 \times 40 \mathrm{~m}$ se sigue realizando con la intención de evaluar la RSA (FIFA, 2016). La habilidad de resistir la fatiga y mantener un alto rendimiento a lo largo del test es uno de los objetivos clave de los test de esprints múltiples (Glaister, Howatson, Pattison, \& McInnes, 2008). Sin embargo, con una recuperación de 90 segundos no se observó 
un decremento significativo $(\mathrm{p}=0.90)$ en el tiempo de esprint (Weston, Castagna, Helsen \& Impellizzeri, 2009). En cuanto a la distancia de ejecución, $40 \mathrm{~m}$ parece ser excesiva, teniendo en cuenta que durante un encuentro, las acciones de alta intensidad tienen una duración de 2-3 segundos, y raramente superan los 30m de longitud (D’Ottavio \& Castagna, 2001; Krustrup \& Bangsbo, 2001; Krustrup et al., 2009). De acuerdo con Castagna et al. (2007) y Weston et al. (2012), protocolos que incluyan esprints de 20-30m con una recuperación de 20-30 segundos, podrían ser más apropiados para la evaluación de la RSA en árbitros.

Los comités arbitrales autonómicos españoles tienen libertad para seleccionar los tests que consideren oportunos para evaluar a los árbitros no profesionales que ostenten las categorías que se rigen bajo la normativa autonómica. En el caso del Comité Técnico de Árbitros (CTA) de la Federación de Fútbol de la Comunidad Valenciana (FFCV), la batería de test que utiliza consta de dos pruebas: 6 carreras de velocidad de $40 \mathrm{~m}$ con 90 segundos de recuperación y una carrera a pie de resistencia de $2.000 \mathrm{~m}$. Se tiene constancia de un solo estudio donde se evalúa la prueba de $2000 \mathrm{~m}$. En este trabajo Ruiz et al. (2011) no encontró correlaciones significativas entre el rendimiento en esta prueba y el VO2max $(\mathrm{p}>0.05)$. Nos encontramos de nuevo ante un test de dudosa validez. Por el contrario, ya se ha demostrado que un test de carácter intermitente como el Test Interválico para la Valoración de la Resistencia Específica en Fútbol (TIVRE-F) es más sensible a las mejoras de la condición física durante una pretemporada en jugadores de fútbol que otros tests como el Course Navette, y proporciona más información (GarcíaLópez et al., 2009). Por tanto, puede que el TIVRE-F sea una buena opción para evaluar la condición física de los árbitros de fútbol.

En el presente trabajo se aplicarán en árbitros de fútbol no profesionales dos baterías de evaluación de la condición física, con el objetivo de comparar la batería que actualmente utiliza la FFCV con una nueva propuesta, en un intento de determinar cuál puede ser más específica para este colectivo.

\section{Material y métodos}

\section{Participantes}

Catorce árbitros de campo comenzaron el estudio, uno de ellos lo abandonó por motivos que le impidieron acudir a las sesiones de testeo, y tres por lesión. Por tanto, la muestra final estuvo compuesta por diez participantes $(n=10)$, todos ellos pertenecientes a categorías de fútbol no profesionales, tuteladas por la FFCV (Tercera División, $n=4$; Regional Preferente, $n=6$ ). La media de edad, peso, altura e IMC de los participantes es 22,5 $\pm 1,84$ años, $69 \pm 5,52 \mathrm{~kg}, 177 \pm 5,73$ cm y $22,04 \pm 1,57 \mathrm{~kg} / \mathrm{m}^{2}$. Los árbitros seguían una plani- ficación del entrenamiento tradicional orientada a alcanzar los puntos de máximo rendimiento en los meses de diciembre y abril. La progresión de la carga se caracterizaba por un alto volumen y baja intensidad en los periodos preparatorios, y un descenso del volumen con aumento de intensidad en los periodos cercanos a las pruebas físicas estipuladas por la FFCV. Realizaban entrenamientos de 90 minutos, 2-4 veces por semana. Los contenidos se distribuían equitativamente entre un programa específico de fuerza orientado a la carrera y un programa de carrera a pie. La carga semanal de partidos arbitrados era de 2-3 encuentros cada fin de semana (150-220 minutos), ya que además de dirigir encuentros de su categoría, también arbitraban partidos de fútbol base. Los participantes fueron informados de los procedimientos a los que se iban a someter y posibles riesgos que podían derivarse de éstos, dando su consentimiento voluntario.

\section{Instrumentos}

Para la correcta realización del TIVRE-F se utilizó el software TIVRE-Fútbol 2.0, que marcaba los ritmos de ejecución de manera que los participantes debían alcanzar la siguiente baliza cuando sonaba la seńal acústica. Durante las sesiones de evaluación, los participantes portaban un monitor de frecuencia cardíaca sincronizado con el dispositivo Polar Team Prol. Para analizar el ritmo cardíaco durante la prueba se utilizó la plataforma online del mismo dispositivo. Los participantes fueron filmados durante las pruebas de RSA con una cámara JVC Everio HD modelo GZ-HD620BE, con una frecuencia de imagen de $50 \mathrm{fps}$.

\section{Procedimiento}

Se diseñó un estudio de caso donde todos los árbitros realizaron dos baterías de tests, con el objetivo de analizar el impacto del transcurso de la temporada sobre el rendimiento en ambas baterías. Todos los procedimientos a los que los árbitros fueron sometidos estuvieron aprobados por el Comité de Ética de la Universidad de Alicante.

Los sujetos acudieron a las sesiones conociendo el procedimiento y objetivos de las pruebas que iban a realizar, estando familiarizados con el protocolo de cada una. Se encontraban en una situación de buena salud. No ingirieron ningún alimento en las dos horas anteriores ni ninguna bebida o sustancia que pudiera alterar el resultado (alcohol, té, café...). No realizaron ejercicio físico en las últimas 48 horas.

Se desarrolló un calentamiento de una duración de $20 \mathrm{mi}$ nutos a realizar antes del inicio de cada sesión: 10' carrera suave, 3' ejercicios de movilidad dinámica, 4’ técnica de carrera y 3' de progresiones. Después del calentamiento se dejó unos minutos antes de comenzar las pruebas en el que los participantes realizaron salidas de velocidad. 
Los árbitros fueron testeados en tres momentos de la temporada, que coincidían con los periodos de máximo rendimiento previstos en la planificación anual: primera semana de septiembre (toma 1, T1), primera semana de diciembre (toma 2, T2) y segunda semana de abril (toma 3, T3). En cada ocasión se realizaron dos baterías de test, siempre en el mismo orden y con un descanso de 48 horas entre ambas.

La primera batería estaba compuesta por las pruebas que la FFCV utiliza actualmente para testear a sus árbitros. Éstas se realizaron en las mismas condiciones que establece la FFCV: en pista atlética de $400 \mathrm{~m}$, con superficie de tartán, no se permitieron tacos que mejoraran la adherencia a la pista $y$ con una separación de 6-8 minutos entre ambas. En orden de ejecución, son las siguientes:

$R S A$ : 6 series de $40 \mathrm{~m}$, con una recuperación de 90 segundos entre ellas (6x40m/90”). La carrera comienza con 1,5m de distancia de la línea de salida.

Resistencia: carrera de 2000 m. La salida se realizó de forma colectiva desde la línea de meta y atendiendo a la señal acústica que implicaba el inicio de la prueba, de igual forma que en las pruebas oficiales.

La segunda batería consistía en la ejecución de las pruebas propuestas por los autores. Se seleccionaron protocolos que ya han sido validados para la evaluación de la RSA y la condición aeróbica en futbolistas (Chaouachi et al., 2010; Rodríguez-Fernández, 2016), considerándose potencialmente válidos en árbitros, dadas que las semejanzas en las demandas físicas y fisiológicas entre árbitros y jugadores de fútbol (Castagna et al., 2007; Reilly \& Gregson, 2006; Stølen et al., 2005; Weston et al., 2011). Estas pruebas se realizaron en un campo de fútbol, con calzado de tacos y dejando una separación de 6-8 minutos entre cada una. Por orden de ejecución, son las siguientes:

$R S A$ : 7 series de $30 \mathrm{~m}$, con una recuperación de 25 segundos entre ellas ( $7 \times 30 \mathrm{~m} / 25$ ”). La carrera comienza con $1,5 \mathrm{~m}$ de distancia de la línea de salida.

Resistencia aeróbica: Test Interválico para la Valoración de la Resistencia Específica en Fútbol (TIVRE-F). Esta prueba tiene las características de un test intermitente incremental: cada árbitro se encuentra en una baliza para dar inicio al test, tras realizar dos vueltas al recorrido que se muestra en la Figura 1 se completa un estadio, entre estadios se establece un periodo de recuperación de 30 segundos, la velocidad aumenta en $0,6 \mathrm{~km} / \mathrm{h}$ entre estadios, la velocidad de inicio es 7,8 $\mathrm{km} / \mathrm{h}$. El test se daba por finalizado cuando al árbitro no llegaba a tiempo a dos balizas sucesivas.

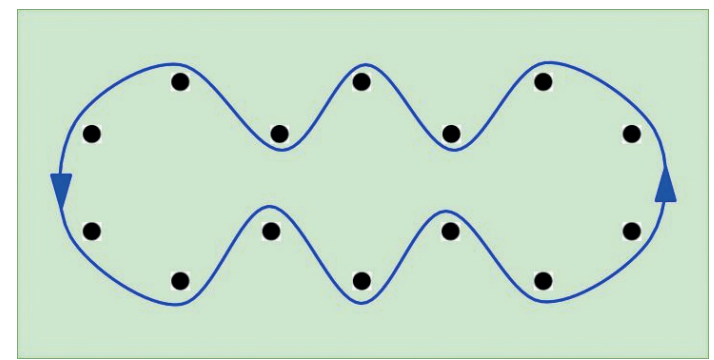

Figura 1. Posición de las balizas y recorrido del TIVRE-F, con una distancia de $9,15 \mathrm{~m}$ entre una baliza y la siguiente (RodríguezFernández, 2016).

\section{Análisis de los datos}

La filmación de las pruebas de esprints repetidos fue posteriormente analizada con el software Kinovea. El tiempo de ejecución se estableció en base al tiempo que tardó el sujeto en cruzar la baliza de salida y de llegada. De estas pruebas se obtuvo el tiempo medio de esprint, el mejor tiempo y el índice de fatiga (Fitzsimons, Dawson, Ward y Wilkinson, 1993). De la carrera a pie de $2000 \mathrm{~m}$ se recogió el tiempo de ejecución, la VAM, Frecuencia Cardiaca Máxima (FCmáx) y Frecuencia Cardiaca Media (FCmedia). Del TIVRE-F se obtuvo la VAM, la Velocidad del Umbral Anaeróbico (V-UAn) de forma manual, de la misma forma que García-López et al. (2003), FCMmáx y porcentaje de recuperación de la FC entre estadios. Entre las diferentes tomas de datos (T1, T2 y T3) se calcularon los porcentajes de variación.

Los resultados se muestran como media y desviación estándar de la media. El tratamiento gráfico de los datos se realizó en el software Microsoft Excel 2010 y el estadístico con el programa IBM SPSS Statistics versión 24.0. Se analizó la normalidad de los datos con la prueba Kolmorov-Smirnov (K-S) y se realizó un análisis de varianza (ANOVA) de medidas repetidas para comparar los resultados entre las distintas tomas y entre grupos. La significación estadística se estableció en $\mathrm{p}<0,05$.

\section{Resultados}

Los datos obtenidos de las pruebas de esprints repetidos se muestran en la tabla Tabla 1 y 2 .

Tabla 1. Resultados de la prueba $6 \times 40 \mathrm{~m} / 90$ ” en la toma 1 (T1), toma 2 (T2) y toma 3 (T3).

\begin{tabular}{cccc}
\hline & $\begin{array}{c}\text { Media de } \\
\text { esprint }(\mathrm{s})\end{array}$ & $\begin{array}{c}\text { Mejor tiem- } \\
\text { po }(\mathrm{s})\end{array}$ & $\begin{array}{c}\text { Índice de } \\
\text { fatiga (\%) }\end{array}$ \\
\hline T1 & $5,66 \pm 0,21$ & $5,47 \pm 0,19$ & $3,42 \pm 2,10$ \\
T2 & $5,69 \pm 0,24$ & $5,55 \pm 0,24$ & $2,58 \pm 2,22$ \\
T3 & $5,75 \pm 0,27$ & $5,62 \pm 0,27$ & $2,30 \pm 1,44$ \\
\hline Variación T1-T3 (\%) & 1,59 & 2,71 & $-32,77$ \\
\hline
\end{tabular}

${ }^{*} \mathrm{p}<0.05$ con respecto a $\mathrm{T} 1$. 
Tabla 2. Resultados de la prueba $7 \times 30 \mathrm{~m} / 25$ ” en la toma 1 (T1), toma 2 (T2) y toma 3 (T3).

\begin{tabular}{cccc}
\hline & $\begin{array}{c}\text { Media de } \\
\text { esprint }(\mathrm{s})\end{array}$ & $\begin{array}{c}\text { Mejor tiempo } \\
(\mathrm{s})\end{array}$ & $\begin{array}{c}\text { Índice de fatiga } \\
(\%)\end{array}$ \\
\hline T1 & $4,70 \pm 0.20$ & $4,56 \pm 0,19$ & $2,98 \pm 1,12$ \\
T2 & $4,76 \pm 0,15$ & $4,62 \pm 0,20$ & $3,01 \pm 1,61$ \\
T3 & $4,81 \pm 0,28$ & $4,66 \pm 0,26$ & $3,20 \pm 1,44$ \\
\hline Variación & 2,27 & 2,04 & 7,57 \\
T1-T3 (\%) & & & \\
\hline
\end{tabular}

${ }^{*} \mathrm{p}<0.05$ con respecto a $\mathrm{T} 1$

Los datos obtenidos en las pruebas de resistencia se muestran en las Tabla 3 y 4.

Tabla 3. Resultados de la prueba de $2000 \mathrm{~m}$ en la toma 1 (T1), toma 2 (T2) y toma 3 (T3).

\begin{tabular}{cccc}
\hline & Tiempo (min) & FCmáx (\%) & FCmed (\%) \\
\hline T1 & $7,50 \pm 0,36$ & $97,57 \pm 1,19$ & $92,07 \pm 1,95$ \\
T2 & $7,32 \pm 0,42$ & $97,60 \pm 2,73$ & $92,24 \pm 3,38$ \\
T3 & $7,57 \pm 0,30$ & $97,93 \pm 3,14$ & $92,96 \pm 2,76$ \\
\hline
\end{tabular}

${ }^{*} \mathrm{p}<0.05$ con respecto a $\mathrm{T} 1$

Tabla 4. Resultados de la prueba TIVRE-F en la toma 1 (T1), toma 2 (T2) y toma 3 (T3).

\begin{tabular}{cccc}
\hline & VAM $(\mathrm{km} / \mathrm{h})$ & V-Uan $(\mathrm{km} / \mathrm{h})$ & FCmáx $(\%)$ \\
\hline T1 & $14,70 \pm 0,86$ & $12,60 \pm 0,57$ & $96,94 \pm 1,80$ \\
T2 & $14,88 \pm 0,74$ & $12,72 \pm 0,68$ & $97,39 \pm 2,29$ \\
T3 & $15,42 \pm 0,80^{*}$ & $13,02 \pm 0,75$ & $97,90 \pm 2,63$
\end{tabular}

${ }^{*} \mathrm{p}<0.05$ con respecto a $\mathrm{T} 1$

El porcentaje de recuperación de la FC entre estadios se muestra en la Figura 2. Los porcentajes de mejora de los tests de resistencia entre las diferentes tomas se muestran en la Figura 3.

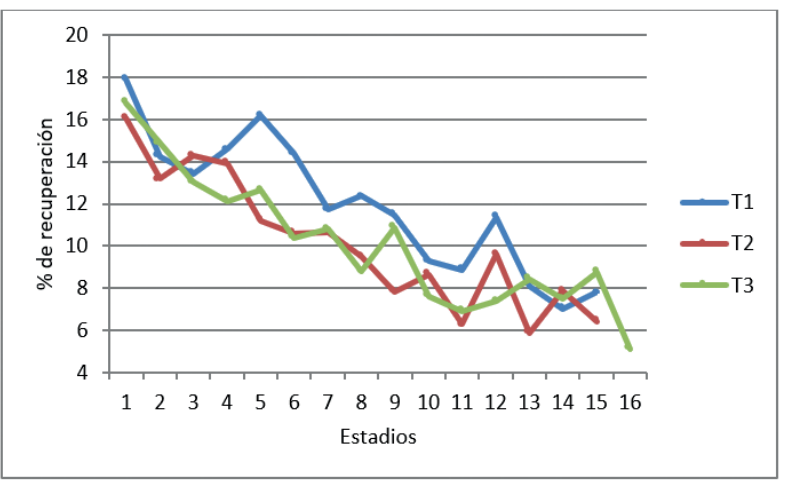

Figura 2. Porcentaje de recuperación de la FC en el test TIVRE-F en la toma 1 (T1), toma 2 (T2) y toma 3 (T3).

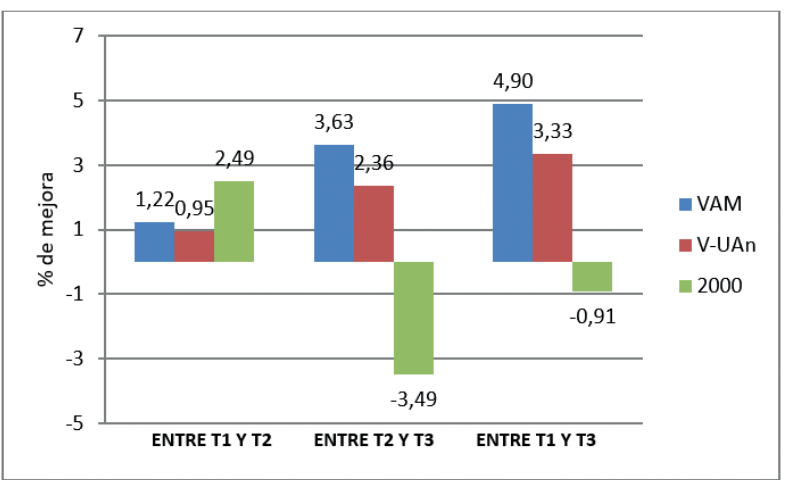

Figura 3. Porcentaje de variación de la VAM y V-UAn en el test TIVRE-F y el tiempo de ejecución en la carrera de $2000 \mathrm{~m}$ en en la toma 1 (T1), toma 2 (T2) y toma 3 (T3).

Se encontró una interacción $(\mathrm{p}=0.011)$ entre el comportamiento de la VAM durante la temporada y el test de resistencia con el que se evaluaba. La Figura 4 muestra este comportamiento en los diferentes tests.

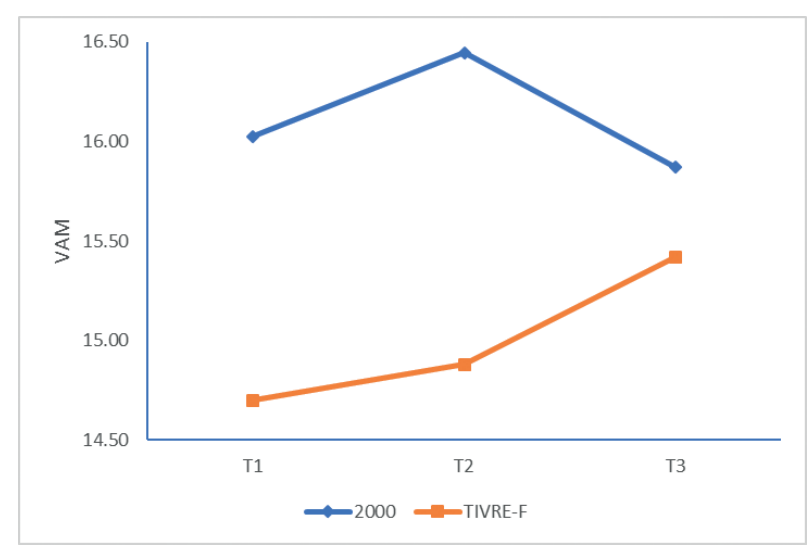

Figura 4. VAM $(\mathrm{m} / \mathrm{s})$ obtenida en los test de resistencia en la toma 1 (T1), toma 2 (T2) y toma 3 (T3).

\section{Discusión}

El objetivo del presente trabajo era la de comparar los test utilizados por la FFCV para evaluar a sus árbitros con una nueva propuesta para intentar determinar cuál puede ser más específica para este colectivo.

La interacción encontrada en los test de resistencia nos dice que ambos test miden cualidades físicas diferentes, ya que el comportamiento del rendimiento en cada uno de ellos describió una curva desigual respecto a otro. Arbitrar es una actividad predominantemente aeróbica por su duración, correspondiéndose al $81 \%$ del consumo máximo de oxígeno (VO2max) (Krustrup \& Bangsbo, 2001). Por tanto, la evaluación de la cualidad aeróbica en este colectivo tiene que ir dirigida a la determinación de la VAM, indicador del VO2 máx y predictor del rendimiento en los deportes intermiten- 
tes (Barbero-Álvarez, 2010). Esteve, Cejuela, \& Menéndez (2010) establecen que el tiempo máximo que la VAM puede ser sostenida es de 6 minutos en sujetos muy entrenados. Por tanto, la velocidad media de la carrera de $2000 \mathrm{~m}$ difícilmente puede representar la VAM de los árbitros, que tardaron más de 7 minutos en realizarla. Con los datos obtenidos, concluimos que esta prueba se realiza por debajo del VO2max $(\approx 92 \%$ FCM). Por el contrario, el TIVRE-F es un test que determina la VAM de forma directa y la V-UAn de forma indirecta (García-López et al., 2003).

Dadas las características metodológicas del test, el TIVRE-F es más específico que la carrera de 2000 m en la evaluación de la condición aeróbica en árbitros de fútbol. Para comenzar, el primero se realiza con el calzado que los árbitros usan durante sus partidos, en el terreno donde se juegan. Además, los cambios de dirección simulan las diferentes trayectorias que siguen los árbitros en el campo, donde cambian de actividad cada 4,3segundos (Krustrup \& Bangsbo, 2001). En cambio, el modo de carrera de la prueba de $2000 \mathrm{~m}$ incluye mantener una velocidad constante o ligeramente variable y desplazarse en una pista donde no se presenta ningún cambio de dirección. Se ha demostrado que el desempeño en un test CODA (Change Of Direction Ability) discrimina a los árbitros por niveles (Yanci, Los Arcos, Grande, \& Casajús, 2016). Por tanto, la necesidad de implementar estos elementos en las baterías de evaluación de este colectivo podría resultar obvia. Los árbitros amateurs, pertenecientes a las categorías tuteladas por la FFCV, no tienen pruebas de estas características.

En el presente trabajo no se observó una mejora en la recuperación de la FC entre estadios del TIVRE-F, como sí se observó en otros estudios (García-López et al., 2009). Este trabajo se realizó en jugadores de un alto nivel competitivo, para valorar los efectos de la pretemporada. De estos datos podemos hipotetizar que el estímulo de entrenamiento semanal al que se someten los árbitros no es suficiente para provocar adaptaciones en este parámetro, ya que estos objetivos sí se lograron en jugadores de fútbol, en un momento en el que la preparación física tiene un papel importante, como es la pretemporada. Por tanto, el TIVRE-F podría tener la capacidad de discriminar a los deportistas por niveles competitivos según su volumen de entrenamiento, a través de la mejoría del porcentaje de recuperación de la FC entre estadios.

A pesar de que la planificación de entrenamiento anual estaba dirigida a obtener el máximo rendimiento en los meses de diciembre y abril, los árbitros no mejoraron su ejecución en la carrera a pie de $2000 \mathrm{~m}$. Sin embargo, el TIVRE-F consiguió recoger mejoras significativas en la VAM durante la temporada. Ya que los contenidos de entrenamiento no se dirigían a aumentar el rendimiento en actividades intermitentes de intensidad variable, puede que esta mejora en la VAM venga dada por la mayor transferencia de los estímulos que los árbitros realizan durante sus encuentros al rendimiento en la prueba. Por tanto, podría existir una tendencia a que los árbitros de fútbol realizan, durante la temporada, actividades que tienen mayor orientación al rendimiento en pruebas aeróbicas de carácter intermitente. Futuras investigaciones podrían dirigirse a establecer una relación directa entre el rendimiento en el TIVRE-F y las actividades realizadas dentro terreno de juego.

Aunque el nivel de nuestros árbitros se asemeja a los ya reportados en la literatura (Sánchez-García, Sánchez-Sánchez, Rodríguez-Fernández, Solano \& Castillo, 2018), el rendimiento en las pruebas de RSA fue decreciendo a lo largo de la temporada. Los árbitros fueron cada vez más lentos en sus carreras de 30 y 40 metros, tanto en parámetros de velocidad máxima como velocidad media. Una posible explicación, vendría dada por una mala planificación del entrenamiento, donde no se entrenó la capacidad de realizar esfuerzos repetidos a alta intensidad, siendo insuficientes los estímulos a los que los árbitros se sometieron durante sus encuentros.

El índice de fatiga en las carreras de $30 \mathrm{~m}$ fue cercano al $3 \%$. Un estudio realizado con futbolistas registró este valor entre 4-6\% para el mismo protocolo (Chaouachi et al., 2010). No obstante, el tiempo medio de esprint fue más elevado en nuestros árbitros. Esto puede deberse a que los árbitros evaluados no son tan rápidos en estas distancias, pero son más resistentes a la aparición de la fatiga. Una segunda hipótesis viene de la preocupación de que los árbitros tienden a no dar el máximo durante las pruebas de evaluación. Podría entenderse que la intensidad del esfuerzo estuvo por debajo de las posibilidades, pudiendo mantener una velocidad más uniforme a lo largo del test y resultar en pérdidas de velocidad inferiores. Ya que la recuperación era muy inferior al protocolo al cual estaban acostumbrados, éstos intentaban evitar los efectos de la fatiga para poder completar tanto la primera, como la segunda prueba. Este comportamiento, en el que se observa una intensidad de esfuerzo submáxima, podría verse también en los test oficiales, donde la prioridad es superar ambas pruebas (la no superación de las pruebas supone no poder dirigir encuentros) y el objetivo secundario es tener un buen rendimiento en ellas.

Dado que los tests de RSA registraron variaciones del rendimiento similares durante la temporada, podría afirmarse que ambos tienen el mismo nivel de aplicabilidad en árbitros. Sin embargo, el test de $7 \times 30 \mathrm{~m}$ ha registrado índices de fatiga superiores a la prueba de 6x40 en dos de las tres tomas. Este resultado puede parecer evidente debido a la diferencia en el tiempo de recuperación (25 vs 90 segundos). Por tanto, se debe considerar una reducción en el periodo de recuperación entre esprints del protocolo actual, para dificultar que los árbitros mantengan un rendimiento uniforme, pudiéndose observar la resistencia a la fatiga durante esprints repetidos, objetivo principal de los tests de RSA.

Actualmente, el periodo de recuperación entre esprines se 
ha reducido a 1 minuto para los árbitros internacionales y para aquellos que ostentan la categoría nacional en la RFEF (Comité Técnico de Árbitros de la RFEF, 2019). No obstante, esta disminución se presenta insuficiente para observar un decremento en la velocidad, de acuerdo con los datos de Balsom, Seger, Sjödin, \& Ekblom (1992). Hasta la fecha, no se han investigado los efectos del entrenamiento de alta intensidad en el rendimiento de la RSA en este colectivo. A este respecto, parece un elemento clave introducir entrenamientos de estas características, puesto que los resultados obtenidos muestran un déficit en la velocidad y mantenimiento de la velocidad durante esprints repetidos a lo largo de una temporada.

\section{Conclusiones}

La carrera de $2000 \mathrm{~m}$ y el TRIVRE-F evalúan cualidades físicas distintas. Esto vendría explicado por las diferencias metodológicas entre ambas pruebas. El TIVRE-F es capaz de registrar variaciones del rendimiento a lo largo de una temporada, obteniendo mejoras significativas en una variable objetivo de las baterías de evaluación en árbitros, la VAM. Además, el TIVRE-F adapta sus características al comportamiento de los árbitros dentro del terreno de juego.

Por otro lado, el test de RSA propuesto registró mayores pérdidas de velocidad a lo largo de la prueba, lo que proporciona más información sobre la capacidad de realizar esprints repetidos. Aunque el número y la distancia ideal de cada esprint sigue siendo una incógnita, se debe considerar una reducción del tiempo de recuperación en el protocolo de RSA actual.

Con este trabajo se han obtenido nuevos datos sobre la idoneidad de las pruebas que la FFCV utiliza en sus árbitros. La batería de test propuesta podría ajustarse en mayor medida a las necesidades de evaluación. No obstante, futuras investigaciones deberán dirigirse a establecer una relación directa entre el rendimiento en las pruebas TIVRE-F y RSA y las actividades realizadas dentro terreno de juego, para confirmar su validez.

\section{Limitaciones}

Una de las limitaciones metodológicas de este estudio viene dada por el bajo volumen muestral. Se presentaron muchas dificultades a la hora de reclutar participantes por cuestiones demográficas, ya que los árbitros pertenecientes a las categorías Regional Preferente y Tercera División están distribuidos no homogéneamente por toda la Comunidad Valenciana. La muestra original constituía un $10 \%$ de la población diana. Sin embargo, la muerte experimental fue muy elevada, contando un total de cuatro bajas, tres de ellas por lesión. A consecuencia, el bajo tamańo muestral ha podido influir en los análisis estadísticos, poniendo en duda la robustez del uso de tests paramétricos.

Otro aspecto que considerar es que en todas las tomas de datos se mantuvo el mismo orden de ejecución de las dos baterías de test. El primer día se ejecutaba la batería que utiliza la FFCV, y el segundo la nueva propuesta. Un diseño más acertado habría consistido en contrabalancear la muestra en dos grupos. Uno de ellos realizaría la Batería 1 el primer día y la Batería 2 el segundo. El segundo grupo seguiría el orden inverso. De esta forma se minimizaría el efecto que la realización de una primera prueba pudiera tener sobre la segunda. Además, debemos tener en cuenta que realizar las pruebas oficiales aumentaba el estrés de los participantes, aunque no fueran calificativas. Desafortunadamente, esta cuestión no se tuvo en consideración en el momento del desarrollo de la metodología.

\section{Referencias}

1. Balsom, P., Seger, J., Sjödin, B., \& Ekblom, B. (1992). MaximalIntensity Intermittent Exercise: Effect of Recovery Duration. International Journal of Sports Medicine, 13(7), 528-533. https://doi. org/10.1055/s-2007-1021311

2. Barbero-Álvarez, J. C. (2010). Valoración y control del rendimiento en los deportes de equipo. En F. Naclerio. (Ed.) Entrenamiento deportivo: fundamentos y aplicaciones en diferentes deportes. (pp. 229-250) Madrid, España: Panamericana.

3. Castagna, C., Abt, G., \& D’Ottavio, S. (2004). Activity profile of international-level soccer referees during competitive matches. Journal of Strength and Conditioning Research, 18(3), 486-90. https://doi. org/10.1519/1533-4287(2004)18<486:APOISR>2.0.CO;2

4. Castagna, C., Abt, G., \& D’Ottavio, S. (2007). Physiological Aspects of Soccer Refereeing Performance and Training. Sports Med, 37(7), 625-646.

5. Castillo, D., Yanci, J., Casajús, J. A., \& Cámara, J. (2016). Physical fitness and physiological characteristics of soccer referees. Science and Sports, 31(1), 27-35. https://doi.org/10.1016/j.scispo.2015.11.003
6. Cerqueira, M. S., da Silva, A. I., \& Marins, J. C. B. (2011). Analysis of the FIFA's Model of Physical Evaluation Applied to the Soccer Referees. Revista Brasileira De Medicina Do Esporte, 17(18), 425-430.

7. Chaouachi, A., Manzi, V., Wong, D. P., Chaalali, A., Laurencelle, L., Chamari, K., \& Castagna, C. (2010). Intermittent endurance and repeated sprint ability in soccer players. Journal of Strength and Conditioning Research, 24(10), 2663-2669. https://oi.org/10.1519/ JSC.0b013e3181e347f4

8. Comité Técnico de Árbitros de la Real Federación Española de Fútbol (2019). Circular No2: Pruebas médicas y físicas. Recuperado de: http:// www.rfef-cta.com/

9. D’Ottavio, S., \& Castagna, C. (2001). Physiological load imposed on elite soccer referees during actual match play. Journal of Sports Medicine and Physical Fitness, 41(1), 27-32. https:// doi.org/10.1519/1533-4287(2001)015<0167:AOMAIE> 2.0.CO;2

10. Esteve J., Cejuela, R., Menéndez J. (2010). Entrenamiento de la resistencia en deportes cíclicos. En F. Naclerio. (Ed.) Entrenamiento depor- 
tivo: fundamentos y aplicaciones en diferentes deportes. (pp. 171-193) Madrid, España: Panamericana.

11. Fédération Internacionale de Football Association (2016). Fitness test for referees (Men \& Women). Recuperado de: http://footballwest.com.au/

12. Fitzsimons, M,. Dawson, B., Ward, D., Wilkinson, A. (1993) Cycling and running tests of repeated sprint ability. Aust J Sci Med Sport. 25(4), 82-87

13. García-López, J., Rodríguez-Marroyo, J.A., Morante-Rábago, J.C., Moreno, C., Asenjo, H., Rubio Hernández, I., Ávila, M.C., Villa, J.G. (2009). Sensibilidad del test de valoración de la Resistencia específica en el fútbol (TVREF) para evaluar la influencia del entrenamiento de pretemporada en la resistencia y capacidad aeróbica de futbolistas profesionales. En "CD II Congreso Internacional de Deportes de Equipo". Fernández Romero y cols. (Eds.) Ed. Centro de Formación Alto Rendimiento. pp. 1-10.

14. García López, J., Villa, J. G., Rodríguez, J. A., Morante J. C., Álvarez, E., \& Jover, R. (2003). Aplicación de un test de es fuerzo interválico (Test de Probst) para valorar la cualidad aeróbica en futbolistas de la liga española. Apunts Medicina de l'Esport, 77(1), 80-88.

15. Glaister, M., Howatson, G., Pattison, J. R., \& McInnes, G. (2008). The reliability and validity of fatigue measures during multiple-sprint work: an issue revisited. Journal of Strength and Conditioning Research, 22(5), 1597-1601. https://doi.org/10.1519/JSC.0b013e318181ab80

16. Krustrup, P., \& Bangsbo, J. (2001). Physiological demands of top-class soccer refereeing in relation to physical capacity: Effect of intense intermittent exercise training. Journal of Sports Sciences, 19(11), 881-891. https://doi.org/10.1080/026404101753113831

17. Krustrup, P., Helsen, W., Randers, M. B., Christensen, J. F., Macdonald, C., Rebelo, A. N., \& Bangsbo, J. (2009). Activity profile and physical demands of football referees and assistant referees in international games. Journal of Sports Sciences, 27(11), 1167-1176. https://doi. org/10.1080/02640410903220310

18. Mallo, J., Navarro, E., Aranda, J. M. G., \& Helsen, W. (2009). Activity profile of top-class association football referees in relation to fitness-test performance and match standard. Journal of Sports Sciences, 27(1), 9-17. https://doi.org/10.1080/02640410802298227

19. Mallo, J., Navarro, E., García-Aranda, J. M., Gilis, B., \& Helsen, W. (2007). Activity profile of top-class association football referees in rela- tion to performance in selected physical tests. Journal of Sports Sciences, 25(7), 805-813. https://doi.org/10.1080/02640410600778602

20. Reilly, T., \& Gregson, W. (2006). Special populations: The referee and assistant referee. Journal of Sports Sciences, 24(7), 795-801. https://doi. org/10.1080/02640410500483089

21. Rodríguez-Fernández, A. (2016). Relación entre la cualidad aeróbica, la recuperación al esfuerzo y la resistencia a la velocidad en futbolistas: influencia del tipo de entrenamiento y del desentrenamiento (Tesis doctoral). Universidad de León, España.

22. Ruiz, J.A., Brito, E., García-Aranda, J.M, Mallo, J., Helsen, W., Sarmiento, S., Navarro, M., García-Manso, J.M. (2011) Physiological profile of national-level Spanish soccer referees. International SportMed Journal, 12(2), 85-91.

23. Sánchez-García, M., Sánchez-Sánchez, J., Rodríguez-Fernández, A., Solano, D., \& Castillo, D. (2018). Relationships between Sprint Ability and Endurance Capacity in Soccer Referees. Sports, 6(2), 28. https://doi. org/10.3390/sports6020028

24. Schenk, K., Bizzini, M., \& Gatterer, H. (2018). Exercise physiology and nutritional perspectives of elite soccer refereeing. Scandinavian Journal of Medicine \& Science in Sports, 28(3), 782-793. https://doi. org/10.1111/sms.12989

25. Stølen, T., Chamari, K., Castagna, C., \& Wisløff, U. (2005). Physiology of soccer: an update. Sports Medicine (Auckland, N.Z.), 35(6), 501-36.

26. Weston, M., Castagna, C., Helsen, W., \& Impellizzeri, F. (2009). Relationships among field-test measures and physical match performance in elite-standard soccer referees. Journal of Sports Sciences, 27(11), $1177-$ 1184. https://doi.org/10.1080/02640410903110982

27. Weston, M., Castagna, C., Impellizzeri, F. M., Bizzini, M., Williams, A. M., \& Gregson, W. (2012). Science and Medicine Applied to Soccer Refereeing: An Update. Sports Medicine, 42(7), 615-632.

28. Weston, M., Drust, B., \& Gregson, W. (2011). Intensities of exercise during match-play in FA Premier League referees and players. Journal of Sports Sciences, 29(5), 527-532. https://doi.org/10.1080/02640414.2 010.543914

29. Yanci, J., Los Arcos, A., Grande, I., \& Casajús, J. A. (2016). Change of direction ability test differentiates higher level and lower level soccer referees. Biology of Sport, 33(2), 173-177. https://doi. org/10.5604/20831862.1198637 